\section{Seguimiento y evaluación basado en resultados en los procesos de matrícula del Sistema Único de Matrícula de la UNMSM}

Monitoring and evaluation based on results in the processes of registration of the Universal Registration System of the

UNMSM

\section{RESUMEN}

El valor de esta investigación es proponer el seguimiento y evaluación basado en resultados en los procesos de matrícula de la SUM-UNMSM, considerando el plan estrategico institucional 2017-2019 de la UNMSM, aprobado mediante Resolución Rectoral N ${ }^{\circ} 001174-\mathrm{R}-17$ de fecha 13 de enero del 2017; el cual requiere un liderazgo político coherente y una fuerte voluntad técnica. Lo opuesto es seguir con acciones rutinarias que provocan insatisfacción en los servicios a los alumnos y padres de familia. El objetivo es proponer los procedimientos basados en valores en el seguimiento y evaluación de resultados en los procesos de matrícula del SUM.

Palabras claves: Sistema único de inscripción; seguimiento y evaluación basado en resultados; indicadores; medición.

\begin{abstract}
The deliverable value of this research is to propose monitoring and evaluation based on results in the registration processes of the SUM-UNMSM, considering the institutional strategic plan 2017-2019 of the UNMSM, approved by Rectoral Resolution No. 001174-R-17 of January 13, 2017, which requires a coherent political leadership and a strong technical will, the opposite is to continue with routine actions that cause dissatisfaction in services to students and parents. The objective is to propose value-based procedures in the monitoring and evaluation based on results in the enrollment processes of the SUM.

Keywords: Single System of Enrollment; Monitoring and evaluation based on results; indicators; measurement.
\end{abstract}

\section{Kennedy Narciso Gómez knarcisog@unmsm.edu.pe \\ Universidad Nacional Mayor de San Marcos, Facultad de Ciencias Administrativas}

(C) Los autores. Este artículo es publicado por Gestión en el Tercer Milenio de la Facultad de Ciencias Administrativas de la Universidad Nacional Mayor de San Marcos. Este es un artículo de acceso abierto, distribuido bajo los términos de la licencia Creative Commons Atribucion- No Comercia_Compartir Igual 4.0 Internacional. (http://creativecommons.org/licenses/by-nc-sa/4.0/) que permite el uso no comercial, distribución y reproducción en cualquier medio, siempre que la obra original sea debidamente citada. 


\section{INTRODUCCIÓN}

Siendo referente de las universidades públicas del Perú cumpliendo la Ley Universitaria $\mathrm{N}^{\circ} 30220$, el 26 de julio del 2016, por primera vez en casi 500 años de vida institucional; el Dr. Orestes Cachay Boza, asumió el cargo de Rector de la Universidad Nacional Mayor de San marcos, tras ser elegido en votación universal, directa y secreta.

De acuerdo al Capítulo VII, artículo $\mathrm{N}^{\circ} 229$ del Estatuto de la UNMSM, dice lo siguiente: la planeación estratégica impulsa la gestión del cambio acorde con las disposiciones que establece el CEPLAN (Centro Nacional de planeamiento Estratégico) y la Ley Universitaria $\mathrm{N}^{\circ} 30220$; $y$, en el artículo $\mathrm{N}^{\circ}$ 230 Señala que: la administración orienta su actividad en todos sus niveles bajo el enfoque de la gestión por resultados; $y$, en el artículo $\mathrm{N}^{\circ}$ 231: conceptualiza sobre rendición de cuentas, ética pública, transparencia como principios de la gestión administrativa. Impulsada por el gobierno nacional a través del Ministerio de Economía y Finanzas; finalmente, el objetivo de la investigación, siguiendo el lineamiento de acción estratégica, es analizar la viabilidad del Seguimiento y Evaluación Basado en Resultados en los procesos de matrícula del SUM-UNMSM, y los objetivos específicos fijados son los siguientes: Analizar el proceso de matrícula semestral y anual de las facultades e implementar acciones de mejora en el SUMUNMSM.

En el Boletín Informativo $\mathrm{N}^{\circ} 42$ del SUM, elaborado por la Oficina de Estadística del Sistema Único de Matricula de la UNMSM, apreciamos la evolución anualizada de alumnos de pregrado matriculados por facultades en los periodos 2015-I. (32,217alumnos matriculados); 2015-II $\quad(26,691$ alumnos matriculados $)$ y 2016-I (31,914 alumnos matriculados). Como es de verse, la disminución de matrícula de estudiantes responde a diversos factores. Uno de ellos es la carga del mercado laboral y el cruce en la programación académica de los horarios en sus facultades Véase Cuadro $\mathrm{N}^{\circ} 1$.

Cuadro 1

Número de estudiantes de pregrado matriculados en la UNMSM en los periodos 2015-I, 2015-II y 2016-I

\begin{tabular}{|c|c|c|c|c|c|c|c|}
\hline \multirow{2}{*}{ Facultad } & \multirow{2}{*}{ Escuela } & \multicolumn{2}{|c|}{ Matriculados 2015-I } & \multicolumn{2}{|c|}{ Matriculados 2015-II } & \multicolumn{2}{|c|}{ Matriculados 2016-I } \\
\hline & & Total EAP & Total Fac. & Total EAP & Total Fac. & Total EAP & Total Fac. \\
\hline \multirow{5}{*}{ MEDICINA } & Medicina Humana & 1161 & \multirow{3}{*}{3120} & 572 & \multirow{3}{*}{2273} & 1166 & \multirow{3}{*}{3155} \\
\hline & Obstetricia & 446 & & 374 & & 465 & \\
\hline & Enfermería & 368 & & 324 & & 392 & \\
\hline & Tecnología Médica & 827 & \multirow{2}{*}{$10 \%$} & 697 & \multirow{2}{*}{$8.7 \%$} & 796 & \multirow{2}{*}{$9.9 \%$} \\
\hline & Nutrición & 318 & & 306 & & 336 & \\
\hline \multirow{2}{*}{$\begin{array}{l}\text { DERECHO Y } \\
\text { CIENCIA POLÍTICA }\end{array}$} & Derecho & 2109 & 2439 & - & - & 2058 & 2465 \\
\hline & Ciencia Política & 330 & $7.8 \%$ & - & - & 407 & $7.7 \%$ \\
\hline \multirow{8}{*}{$\begin{array}{l}\text { LETRAS Y } \\
\text { CIENCIAS } \\
\text { HUMANAS }\end{array}$} & Literatura & 309 & \multirow{3}{*}{1998} & 298 & 1928 & 307 & \multirow{3}{*}{1955} \\
\hline & Filosofía & 265 & & 246 & & 266 & \\
\hline & Lingüística & 244 & & 232 & & 241 & \\
\hline & Comunicación Social & 521 & \multirow{5}{*}{$6.4 \%$} & 521 & \multirow{5}{*}{$7.4 \%$} & 517 & \multirow{5}{*}{$6.1 \%$} \\
\hline & Arte & 260 & & 241 & & 230 & \\
\hline & $\begin{array}{l}\text { Bibliotecología y Ciencias de } \\
\text { la Información }\end{array}$ & 280 & & 275 & & 268 & \\
\hline & Danza & 33 & & 30 & & 37 & \\
\hline & Conservación y Restauración & 86 & & 85 & & 89 & \\
\hline \multirow{3}{*}{$\begin{array}{l}\text { FARMACIA Y } \\
\text { BIOQUÍMICA }\end{array}$} & Farmacia y Bioquímica & 545 & 780 & 451 & 657 & 529 & 749 \\
\hline & Ciencia de los Alimentos & 114 & \multirow{2}{*}{$2.5 \%$} & 115 & \multirow{2}{*}{$2.5 \%$} & 109 & \multirow{2}{*}{$2.3 \%$} \\
\hline & Toxicología & 121 & & 91 & & 111 & \\
\hline ODONTOLOGÍA & Odontología & 452 & $\begin{array}{c}452 \\
1.5 \% \\
\end{array}$ & 394 & $\begin{array}{c}394 \\
1.5 \% \\
\end{array}$ & 475 & $\begin{array}{c}175 \\
1.5 \% \\
\end{array}$ \\
\hline \multirow[t]{2}{*}{ EDUCACIÓN } & Educación & 1089 & 1628 & 1029 & 1551 & 1090 & 1628 \\
\hline & Educación Física & 539 & $5.2 \%$ & 522 & $6 \%$ & 538 & $5.1 \%$ \\
\hline \multirow{3}{*}{$\begin{array}{l}\text { QUÍMICA E } \\
\text { INGENIERÍA } \\
\text { QUÍMICA }\end{array}$} & Química & 395 & 1238 & 377 & 1185 & 412 & 1251 \\
\hline & Ingeniería Química & 560 & \multirow{2}{*}{$4 \%$} & 547 & \multirow{2}{*}{$4.6 \%$} & 562 & \multirow{2}{*}{$3.9 \%$} \\
\hline & Ingeniería Agroindustrial & 283 & & 261 & & 277 & \\
\hline
\end{tabular}


Seguimiento y evaluación basado en resultados en los procesos de matrícula del Sistema Único de Matrícula de la UNMSM

\begin{tabular}{|c|c|c|c|c|c|c|c|}
\hline \multirow{2}{*}{ Facultad } & \multirow{2}{*}{ Escuela } & \multicolumn{2}{|c|}{ Matriculados 2015-I } & \multicolumn{2}{|c|}{ Matriculados 2015-II } & \multicolumn{2}{|c|}{ Matriculados 2016-I } \\
\hline & & Total EAP & Total Fac. & Total EAP & Total Fac. & Total EAP & Total Fac. \\
\hline $\begin{array}{l}\text { MEDICINA } \\
\text { VETERINARIA* }\end{array}$ & Medicina Veterinaria & 404 & $\begin{array}{c}404 \\
1.3 \%\end{array}$ & - & - & 427 & $\begin{array}{c}427 \\
1.3 \%\end{array}$ \\
\hline \multirow{3}{*}{$\begin{array}{l}\text { CIENCIAS } \\
\text { ADMINISTRATIVAS }\end{array}$} & Administración & 1229 & \multirow{2}{*}{2566} & 1201 & \multirow{2}{*}{2479} & 1233 & \multirow{2}{*}{2513} \\
\hline & Administración de Turismo & 621 & & 613 & & 595 & \\
\hline & Negocios Internacionales & 716 & $8.3 \%$ & 665 & $9.5 \%$ & 685 & \\
\hline \multirow{3}{*}{$\begin{array}{l}\text { CIENCIAS } \\
\text { BIOLÓGICAS }\end{array}$} & Ciencias Biológicas & 340 & 750 & 326 & 707 & 349 & 761 \\
\hline & Genética & 181 & \multirow{2}{*}{$2.4 \%$} & 171 & \multirow{2}{*}{$2.7 \%$} & 174 & \multirow{2}{*}{$2.4 \%$} \\
\hline & Microbiología & 229 & & 210 & & 238 & \\
\hline \multirow{3}{*}{$\begin{array}{l}\text { CIENCIAS } \\
\text { CONTABLES }\end{array}$} & Ciencias Contables & 1172 & 1901 & 1127 & 1811 & 1222 & 2353 \\
\hline & Gestión Tributaria & 354 & \multirow{2}{*}{$6.1 \%$} & 336 & \multirow[b]{2}{*}{$6.97 \%$} & 557 & \multirow[b]{2}{*}{$7.9 \%$} \\
\hline & $\begin{array}{l}\text { Auditoría Empresarial y del } \\
\text { Sector Público }\end{array}$ & 375 & & 348 & & 574 & \\
\hline \multirow{3}{*}{$\begin{array}{l}\text { CIENCIAS } \\
\text { ECONÓMICAS }\end{array}$} & Economía & 1290 & 1906 & 1255 & 1874 & 1165 & 1925 \\
\hline & Economía Pública & 309 & \multirow{2}{*}{$6.1 \%$} & 309 & \multirow{2}{*}{$7.2 \%$} & 382 & \multirow{2}{*}{$6.0 \%$} \\
\hline & Economía Internacional & 307 & & 310 & & 378 & \\
\hline \multirow[b]{2}{*}{ CIENCIAS FÍSICAS } & Física & 337 & 1043 & 308 & 959 & 356 & 1093 \\
\hline & $\begin{array}{l}\text { Ingeniería Mecánica de } \\
\text { Fluidos }\end{array}$ & 706 & $3.4 \%$ & 651 & $3.7 \%$ & 737 & $3.4 \%$ \\
\hline \multirow{4}{*}{$\begin{array}{l}\text { CIENCIAS } \\
\text { MATEMÁTICAS }\end{array}$} & Matemática & 455 & 151 & 415 & 141 & 448 & 1512 \\
\hline & Estadística & 292 & 1017 & 277 & 1710 & 286 & 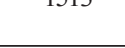 \\
\hline & Investigación Operativa & 469 & $40 \%$ & 449 & 5.40 & 471 & 70 \\
\hline & Computación Científica & 298 & 4.970 & 275 & .470 & 308 & 4.170 \\
\hline & Historia & 301 & & 293 & & 310 & \\
\hline & Sociología & 409 & 2272 & 389 & 2181 & 415 & 2271 \\
\hline CIENCIAS & Antropología & 345 & & 337 & & 364 & \\
\hline SOCIALES & Arqueología & 302 & & 290 & & 300 & \\
\hline & Trabajo Social & 454 & $7.3 \%$ & 434 & $8.4 \%$ & 441 & $7.1 \%$ \\
\hline & Geografía & 461 & & 438 & & 441 & \\
\hline & Ingeniería Geológica & 428 & & 398 & & 401 & \\
\hline INGENIERÍA & Ingeniería Geográfica & 341 & 1641 & 311 & 1548 & 310 & 1674 \\
\hline GEOLÓGICA, & Ingeniería de Minas & 227 & & 206 & & 234 & \\
\hline $\begin{array}{l}\text { MINERA, } \\
\text { METALÚRGICA Y }\end{array}$ & Ingeniería Metalúrgica & 351 & & 344 & & 369 & \\
\hline GEOGRAFÍA & Ingeniería Civil & 256 & $5.3 \%$ & 253 & $6.0 \%$ & 286 & $5.2 \%$ \\
\hline & Ingeniería Ambiental & 38 & & 36 & & 74 & \\
\hline & Ingeniería Industrial & 1185 & & 1098 & & 1186 & \\
\hline $\begin{array}{l}\text { INGENIERÍA } \\
\text { INDUSTRIAL }\end{array}$ & $\begin{array}{l}\text { Ingeniería Textil y } \\
\text { Confecciones }\end{array}$ & 371 & 1596 & 354 & 1491 & 373 & 1646 \\
\hline & $\begin{array}{l}\text { Ingeniería de Seguridad y } \\
\text { Salud en el Trabajo }\end{array}$ & 40 & $5.1 \%$ & 39 & $5.7 \%$ & 87 & $5.2 \%$ \\
\hline & Psicología & 733 & 790 & 633 & 685 & 737 & 647 \\
\hline PSICOLOGÍA & $\begin{array}{l}\text { Psicología Organizacional y } \\
\text { de la Gestión Humana }\end{array}$ & 57 & $2.5 \%$ & 52 & $2.6 \%$ & 110 & $2.7 \%$ \\
\hline & Ingeniería Electrónica & 651 & 1513 & 610 & 1437 & 688 & 1623 \\
\hline INGENIERÍA & Ingeniería Eléctrica & 526 & & 503 & & 555 & \\
\hline ELECTRONICA & $\begin{array}{l}\text { Ingeniería de } \\
\text { Telecomunicaciones }\end{array}$ & 336 & $4.9 \%$ & 324 & $5.5 \%$ & 380 & $5.1 \%$ \\
\hline INGENIERÍA & Ingeniería de Sistemas & 1132 & 1552 & 1032 & 1421 & 1100 & 1590 \\
\hline INFORMÁTICA & Ingeniería de Software & 420 & $5.0 \%$ & 389 & $5.5 \%$ & 490 & $5.0 \%$ \\
\hline & & TOTAL & $\begin{array}{l}32217 \\
100 \%\end{array}$ & TOTAL & $\begin{array}{l}26696 \\
100 \%\end{array}$ & TOTAL & $\begin{array}{l}31914 \\
100 \%\end{array}$ \\
\hline
\end{tabular}

Fuente: Oficina Sistema Único de Matricula - UNMSM. Facultades matriculadas hasta el 30/04/2016. 
El objetivo delSUM ${ }^{1}$ es priorizary optimizar los procesos academicos y administrativos de la matricula vía internet en los programas de pregrado y posgrado de la UNMSM.

El Boletín Informativo del SUM-UNMSM $\mathrm{N}^{\circ} 42$ menciona que hubo un incremento de matrícula vía internet en los semestres 2015I y $2016-$ I, del $64.8 \%$ al $67.2 \%$. Del total de estudiantes matriculados, opinamos que aún los porcentajes son bajos y el proceso de matrícula, vía internet y manual, debe evitar ser crítico, lento, burocrático y actualizar los sistemas informáticos del SUM, a la Nueva Ley Universitaria. Por tanto, consideramos que la UNMSM, debe aplicar un sistema de seguimiento y evaluación basado en los procesos de matrícula; a fin de crear valor agregado en los servicios durante los procesos de matrícula, como consecuencia de lo anterior se plantea la siguiente interrogante: ¿Cuál será el impacto que producirá el desarrollo del seguimiento y evaluación basado en resultados en los procesos de matrícula del Sistema Único de Matrícula de la UNMSM?

La investigación se justifica en las experiencias exitosas de países de la OCDE $(2002)^{2}$, al aplicar las buenas prácticas del sistema de seguimientos y evaluación basado en resultados.

En el libro titulado Modelo de diez pasos para asegurar la implementación de un sistema de seguimiento y evaluación basado en resultados, explica que los expertos están de acuerdo con el primer párrafo del Plan Estratégico del PNUD 2008-2011. Lo siguiente: "mejoras reales en la vida de las personas, y en las opciones y oportunidades que tienen" ${ }^{3}$.

La planificación, el seguimiento y la evaluación se une en (Gestión Basada en Resultados) GBR. La GBR es "una estrategia

\footnotetext{
1 Manual de Organización y Funciones del SUM-UNMSM.

2 Zall K. Jody, Rist. Ray C.,(2004), "Manual para gestores del desarrollo: Diez pasos hacia un sistema de seguimiento $\mathrm{y}$ evaluación basado en resultados", Banco Mundial-Washington.

3 PNUD, (2008), “UNDP Strategic Plan, 2008-2011: Accelerating Global Progress on Human Development", Junta Ejecutiva, Documento DP/2007/43 (en virtud de DP/2007/32).
}

general de gestión cuyo objetivo es lograr un mejor desempeño y resultados demostrables" ${ }^{\prime 4}$.

\section{MÉTODOS}

\section{Método y tipo de investigación}

Empleamos los métodos "inductivo-deductivo", a fin de manejar adecuadamente la información durante el desarrollo de la investigación referida a la variable independiente: "Seguimiento y Evaluación Basado en Resultados" y la variable dependiente: "Proceso de Matricula".

\section{Fórmula de la muestra empleada}

$$
n=\frac{Z^{2} p q N}{e^{2}(N-1)+Z^{2} p q}
$$

\section{Objetivos de la planificación del seguimiento y evaluación basada en resultados del SUM-UNMSM.}

- Utilizar el sistema de seguimiento y evaluación basado en resultados.

- Apoyar la rendición de cuentas semestral y anual sustantiva a los funcionarios. Que se replicaría a jefes de línea de la Universidad.

- Utilizar la información de las Oficinas de Estadísticas e Informáticas de las facultades para la toma de decisiones basada en evidencias de la organización.

- Almacenar sistemáticamente las lecciones aprendidas para mejorar el conocimiento individual y el de la organización.

\section{Explicación estratégica de seguimiento y evaluación basado en resultados en los procesos de matrícula del Sistema Único de Matricula.}

Las autoridades públicas en la Universidad ahora deben prestar atención a los resultados que logran sus administraciones; los alumnos exigen calidad de los servicios que la UNMSM

\footnotetext{
4 UNEG, (2007), "The Role of Evaluation in Results-based Management". Disponible en: http://www.unevaluation.org/ papersandpubs/documentdetail.jsp?
} 
provee. Es importante mencionar que un alumno está oficialmente matriculado en la Universidad Nacional Mayor de San Marcos cuando cumple con los requisitos establecidas por el Nuevo Reglamento de Matricula aprobado según Resolución Rectoral $\mathrm{N}^{\circ} 01163-\mathrm{R}-17$, de fecha 07 de marzo del 2017 y obtenga el reporte de matrícula que será entregado al alumno por la Unidad de Matricula de cada Facultad. Asimismo, señala el reglamento indicado las funciones que deberá cumplir el SUM, que procederálas matriculas dentro del cronograma de actividades académicas aprobado por Resolución Rectoral, el procedimiento de matrícula regular, el procedimiento de rectificación de matrícula, el procedimiento de reactualización de matrícula, el procedimiento del cierre de actas. En el plan estrategico institucional 2017-2019 de la UNMSM, uno de los objetivos estratégicos es mejorar la calidad de la gestión institucional y la acción es implementar la política de modernización de la gestión pública mediante el rediseño de procesos administrativos y académicos con el soporte tecnológico adecuado; asimismo, encontramos la visión del sector educación y la misión institucional que describimos a continuación.

\subsection{Visión del Sector Educación}

Los peruanos acceden a una educación que les permite desarrollar su potencial desde la primera infancia y convertirse en ciudadanos que valoran su cultura, conocen sus derechos y responsabilidades, desarrollan sus talentos y participan de manera innovadora, competitiva y comprometida en las dinámicas sociales, contribuyendo al desarrollo de sus comunidades y del país en su conjunto.

\subsection{Misión Institucional}

"Generar y difundir conocimiento
científico tecnológico, humanístico,
formando profesionales e investigadores
líderes, con valores y respetuosos de la
diversidad cultural, promotores de la
identidad nacional basada en una cultura
de calidad y responsabilidad social para
contribuir al desarrollo sostenible del país
y la sociedad".

\subsection{Visión del Sistema Único de Matricula.}

Ser una oficina que brinde calidad en sus servicios a la comunidad sanmarquina, mediante la integración, comunicación, trabajo en equipo, uniformidad de criterios implementada con recursos humanos eficientes y eficaces, necesarios para el cumplimiento del plan estratégico institucional al 2011.

\subsection{Misión del Sistema Único de Matricula.}

Somos una dependencia responsable de procesar la matrícula de pre y posgrado de la Universidad Nacional Mayor de San Marcos, así como realizar los trámites académicos relacionados a este proceso. Todo esto se lleva a cabo registrando el avance académico, cumpliendo y haciendo cumplir el reglamento general de matrícula, de manera ágil y ordenada, empleando tecnología de avanzada, permitiendo centralizar dicha información con la seguridad necesaria.

\subsection{Valores del Sistema Único de Matricula.}

- Honestidad: Expresada mediante la transparencia en las labores, el respeto a los demás y a nosotros mismos.

- Responsabilidad: Referida al fiel cumplimiento de las tareas encomendadas, realizando las labores, dando lo mejor de nuestras capacidades.

- Objetividad: Emitir las opiniones, dictámenes o informes que reflejan la realidad de los asuntos analizados.

- Cooperación: Reflejada en el apoyo que se brinda a las autoridades $\mathrm{u}$ otras dependencias de la universidad.

\subsection{Objetivo}

Construirlosprocedimientosyorientaciones delegatorio y de gestión basados en valores para el seguimiento y evaluación basado 
en resultados del Nuevo Estatuto inserto al Plan Estrategico Institucional y del Nuevo Reglamento de Matricula.

\subsection{Análisis interno en los procesos de matrícula del Sistema Único de Matrícula de la UNMSM.}

Hemos analizado los procesos críticos de orden estratégico, operativo y de soporte, a fin de tener un mejor entendimiento al respecto. (Véase el Grafico $\mathrm{N}^{\circ} 1$ ).

Proceso 1. "Gestión estratégica" se da por determinadas y cumplimiento por las áreas inferiores.

Proceso 2, 3, 4, 5, 6. "Reporte internet". A través de la Resolución Rectoral se cumple los procesos de matrícula anualizado.

Proceso 7. La matrícula de verano se realiza previa apertura de cursos particular, para lo cual los alumnos cumplen los requisitos del Nuevo Reglamento de Matricula.

\subsection{Identificar Mejora en los procesos de matrícula del Sistema Único de Matricula ${ }^{5}$.}

Proponemos el siguiente proceso mejorado de matrícula a nivel macro para la Universidad Nacional Mayor de San Marcos. Véase el Gráfico $\mathrm{N}^{\circ} 2$.

\section{RESULTADOS}

\section{Propuesta de Seguimiento y Evaluación basado en Resultados en los procesos de matrícula del SUM- UNMSM.}

El seguimiento y evaluación, basada en resultados, son etapas del plan operativo del SUM, inserto al planeamiento estratégico institucional de la Universidad Nacional Mayor de San Marcos; que nos permite identificar los resultados de la administración y la medición del desempeño de los administrados sobre la base de los objetivos estratégicos que deben construirse y ser presentados.

5 Narciso, G. K., (2012) "Rediseño de Procesos para Mejorar la Matricula en la Facultad de Ciencias Administrativas de la Universidad Nacional del Callao", R.R. N990-2012-Callao-Perú

\section{Objetivo}

Construir los procedimientos y orientaciones delegatorio y de gestión, basados en valores para el seguimiento y evaluación en resultados del plan operativo Institucional del SUM inserto al plan estratégico institucional de la UNMSM.

\section{Programa de acciones de} seguimiento y evaluación basado en resultados en los procesos de matrícula del SUM ${ }^{6}$.

3.1. Los decanos, el vicedecano académico, los directores de las Escuelas Académicas Profesionales, la Dirección de Administrativa, el jefe de la Unidad de Matricula y dependencias administrativas involucrados en los procesos de matrícula de pre grado y posgrado de la Universidad Nacional Mayor de San Marcos, emitirán un informe de seguimiento y evaluación de los resultados del desempeño semestral y anual, que dé cuenta de su administración ante el Jefe del SUM, con la finalidad de elaborar, desarrollar, sustentar el informe unificado ante el Consejo Universitario.

3.2. El informe unificado del SUM contendrá lo siguiente: resumen ejecutivo; el problema en el desempeño y los cambios en la estructura organizativa; impactos de los indicadores de medición del desempeño, y análisis de las desviaciones con respecto a los objetivos; actividades y proyectos importantes relacionadas al proceso de matrícula, productos/servicios brindados, recursos programados y ejecutados, evolución de ejecución; proyectos en ejecución, porcentaje del avance físico y financiero, desviaciones y sus razones respecto a las metas físicas y financieras programadas.

3.3. El Jefe del SUM, presentará el informe unificado debidamente detallado de los logros de éxito y las desviaciones o deficiencias incurridas por las áreas

6 Directiva $\mathrm{N}^{\circ}$ 002-14-R., "Seguimiento y evaluación del plan de desarrollo institucional 2014 - 2021 de la Universidad Nacional del Callao" 


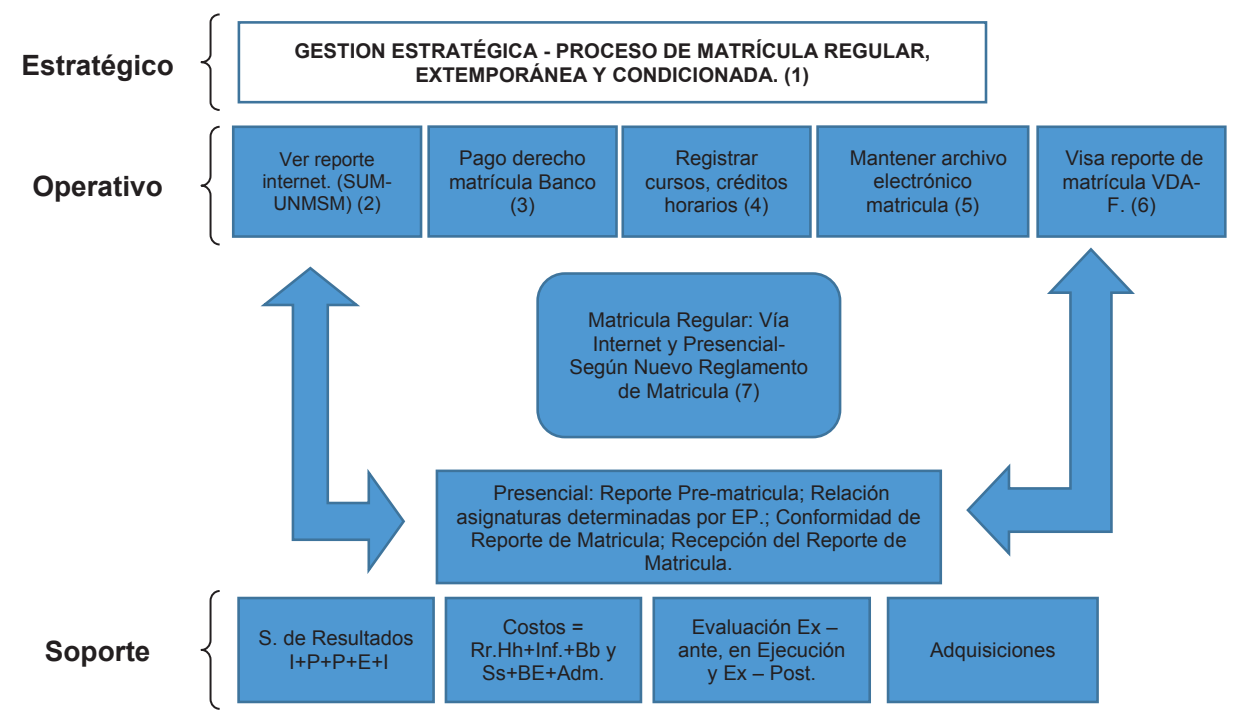

Grafico 1. Análisis Interno - Proceso de Matrícula - SUM-UNMSM.

Fuente: Elaboración propia.

Leyenda: S=Seguimiento, I=Insumo, P=Proceso, P=Producto, E=Efecto, I=Impacto, Rr.Hh=Recursos Humanos, Inf.=Infraestructura, Bb y Ss=bienes y servicios, Adm= administración.

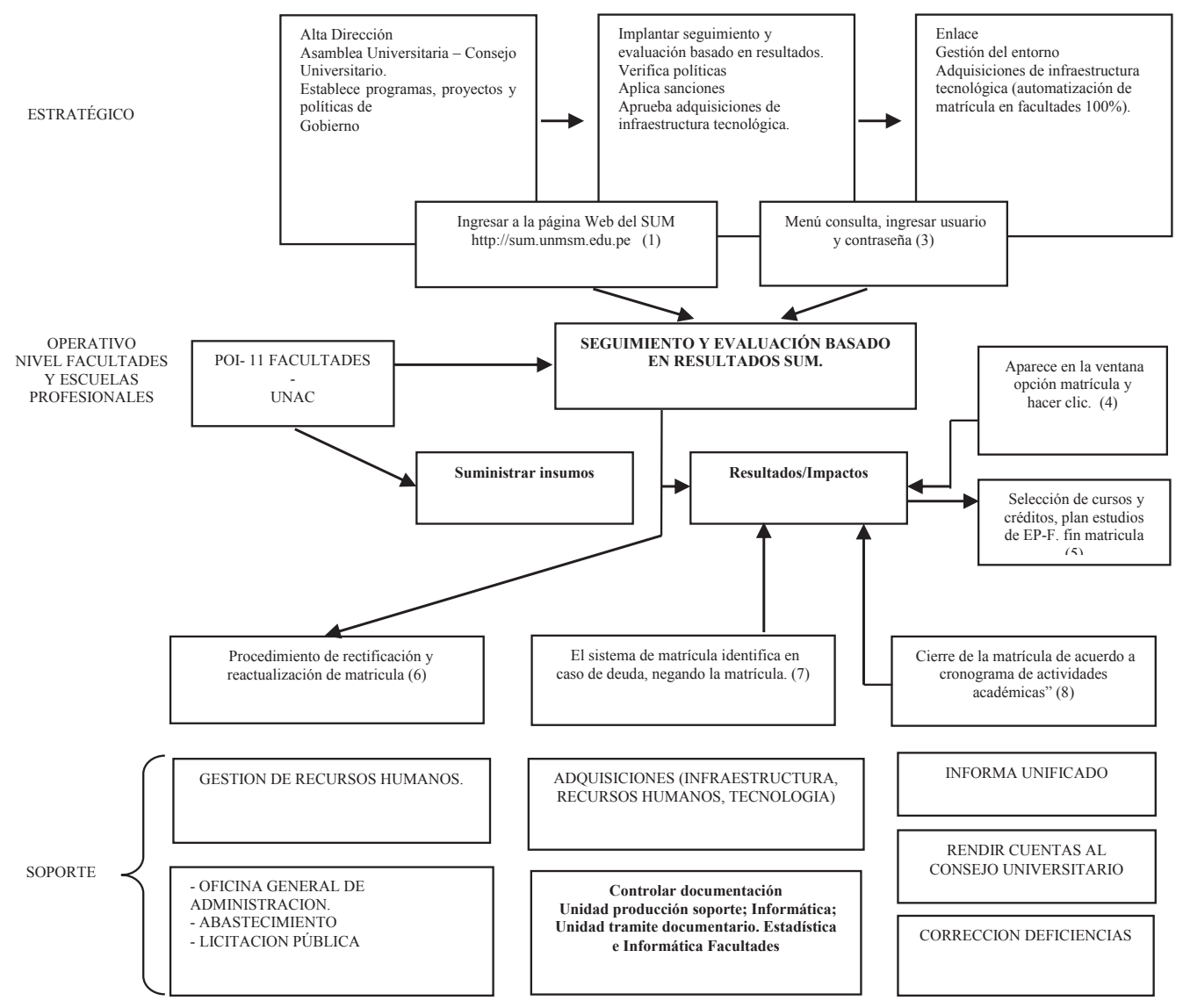

Grafico 2.

Fuente: Elaboración propia. 
respectivas inmersas en el proceso de matrícula.

3.4. El Rector deberá citar a una sesión extraordinarioalConsejoUniversitario, cuya única agenda deberá ser: Presentación y sustentación de informe de seguimiento y evaluación basada en resultados de la Oficina del Sistema Único de Matricula, correspondiente al semestre académico 2017-I.

3.5. El Jefe del SUM deberá sustentar el informe unificado como medio de rendir cuentas del seguimiento $y$ evaluación basada en resultados del proceso de matrícula ante el Consejo Universitario de la UNMSM.

3.6. El informe unificado de seguimiento y evaluación basado en resultados semestral y anual del SUM - UNMSM, serán presentados al Rectorado dentro de los 30 días de vencido el semestre y el año. Las informaciones solicitadas por el Consejo Universitario serán retroalimentadas por el SUM y enviados al área correspondiente, para superar las deficiencias y deberán recibir apoyo logístico y presupuestal sobre el caso, a través de una Resolución Rectoral.

\section{DISCUSIÓN}

La Universidad Nacional Mayor de San Marcos, como referente de las universidades públicas, debería implementar el enfoque de seguimiento y evaluación basado en resultados en los procesos de matrícula del SUM para obtener buenos resultados, mediante una adecuada planificación y, para ello, deberá elaborar un plan estrategico y el plan operativo institucional.

El seguimiento y evaluación basada en resultados, en nuestro caso, son etapas del plan operativo institucional que deberá elaborar la Oficina del Sistema Único de Matricula, inserto al Planeamiento Estratégico Institucional de la Universidad Nacional Mayor de San Marcos, que nos permite identificar los resultados de la administración y la medición del desempeño de los administrados sobre la base de los objetivos estratégicos construidos y presentados en el plan y en el manual del SUM. En este caso, solo anualmente se presentan resultados de las actividades que se unifican en el rectorado para presentar la memoria anual de la institución. Estas acciones generan y seguirán generando desventajas ante nuestros competidores en el sector de educación superior.

El SUM deberá actualizar sus normas y directivas internas acordes al Nuevo Estatuto de la UNMSM y a la Nueva Ley Universitaria. Sugerimos su inmediata actualización.

El SUM debe actualizar el hardware y software para brindar efectividad en el servicio y no sea vulnerable en el acceso de actas de notas.

\section{CONCLUSIONES:}

1. De acuerdo con las discusiones de la investigación y los resultados obtenidos, el SUM-UNMSM tiene alto valor de desempeño que debemos mantener $\mathrm{y}$ seguir fortaleciéndole. Sugerimos la implementación del enfoque de seguimiento y evaluación, basado en resultados en los procesos de matrícula del SUM.

2. Sehanidentificado queelSUM carece delplan operativo y plan estratégico institucional; su elaboración permitirá identificar los resultados delaadministraciónyla medición del desempeño de los administrados, sobre la base de los objetivos estratégicos construidos y presentados en el plan y en el manual del SUM.

3. Se identificó la falta de actualización del Sistema Único de Matricula referente a los cambios del entorno externo: Sugerimos actualizar sus normas y directivas internas acordes al Nuevo Estatuto de la UNMSM y a la Nueva Ley Universitaria.

4. Se identificaron las siguientes acciones: El SUM debe actualizar el hardware y software para brindar efectividad en el servicio y no sea vulnerable en el acceso de actas de notas.

\section{REFERENCIAS BIBLIOGRÁFICAS:}

CHIAVENATO, I. Administración de Recursos Humanos. Introducción a la Teoría General de la Administración.

Directiva $\mathrm{N}^{\circ}$ 002-14-R., (2014) "Seguimiento y evaluación del plan de desarrollo institucional 
2014 - 2021 de la Universidad Nacional del Callao"

Hernández R., Fernández C., Baptista P., (2014) "Metodología de la Investigación", Mc Graw Hill, sexta edición, México.

Manual de Organización y Funciones, (2014) Oficina de Registros de Archivos Académicos-UNACPerú.

NARCISO, K. (2012) "Rediseño de Procesos para Mejorar la Matricula en la Facultad de Ciencias Administrativas de la Universidad Nacional del Callao". Informe Final VRI-UNAC. Callao-Lima.

Programa de las Naciones Unidas para el Desarrollo, (2009), "Manual de planificación, seguimiento y evaluación de los resultados de desarrollo", NY-EE.UU.
PNUD, (2008), “UNDP Strategic Plan, 2008-2011: Accelerating Global Progress on Human Development", Junta Ejecutiva, Documento DP/2007/43 (en virtud de DP/2007/32).

TOLEDO, Emerson. Tesis "Rediseño de procesos para la mejora del otorgamiento de créditos en una empresa de micro finanzas", UP, 2007.

UNEG, (2007), "The Role of Evaluation in Resultsbased Management". Disponible en: http:// www.unevaluation.org/papersandpubs/ documentdetail.jsp?

Zall K., Jody., Rist., y Ray C., (2004), "Manual para gestores del desarrollo: Diez pasos hacia un sistema de seguimiento y evaluación basado en resultados", Banco Mundial-Washington. 
Kennedy Narciso Gómez 